\title{
Association of polymorphism in glutathione S-transferase loci with susceptibility and outcome in rheumatoid arthritis: comparison with the shared epitope
}

\begin{abstract}
Derek L Mattey, Andrew B Hassell, Michael Plant, Peter T Dawes, William R Ollier, Peter W Jones, Anthony A Fryer, Julie E Alldersea, Richard C Strange
\end{abstract}

Staffordshire Rheumatology Centre, The Haywood, High Lane, Burslem, Stoke on Trent, Staffordshire ST6 7AG

D L Mattey A B Hassell M Plant

P T Dawes

ARC Epidemiology Research Unit, Manchester University, Manchester W R Ollier

Department of Mathematics, Keele University, Staffordshire $\mathrm{P} W$ Jones

Clinical Biochemistry Research Laboratory, School of Postgraduate Medicine, Keele University, North Staffordshire Hospital, Stoke on Trent, Staffordshire

A A Fryer

J E Alldersea

R C Strange

Correspondence to: Dr D L Mattey.

Accepted for publication 31 December 1998
Objective-To determine whether glutathione S-transferase GSTM1, GSTM3, GSTT1, and GSTP1 genotypes influence susceptibility or outcome in rheumatoid arthritis (RA).

Methods-277 RA patients were compared with 577 controls to examine any associations between GST genotypes and susceptibility to RA. The effect of genotypes on outcome (Larsen and functional scores) and time integrated acute phase responses (erythrocyte sedimentation rate and $\mathrm{C}$ reactive protein) was assessed in 122 patients with disease duration of 5-10 years. GST and HLA-DRB1 genotypes were determined using polymerase chain reaction based assays. Data were analysed using multiple regression analysis with correction for age, sex, disease duration, and the DRB1 associated shared epitope (SE) and rheumatoid factor (RF) positivity where appropriate.

Results-The GSTM $1{ }^{\star} A{ }^{*} B$ genotype was less common in RA cases ( 3 of 276) than in controls ( 22 of 591) (exact $p=0.047)$, though significance was lost when adjustment was made for multiple comparisons. The Larsen score was higher $(p=0.039)$ in the (8).9) than those with GSTM1 genotypes (74.7), and this was independent of the SE. Again, correction for multiple testing resulted in loss of significance. The difference in Larsen scores between patients homozygous or negative for the SE (87.9 $v 74.3)$ was similar to that between GSTM1 null and non-null patients. No associations between GSTM3 or GSTT1 genotypes and disease markers were identified although the association between $G S T P 1^{\star} B I^{\star} B$ and Larsen score approached significance $(p=0.096)$.

Conclusion-It is proposed that certain GSTs may influence susceptibility and radiological progression in $R A$ and that this is independent of the effect of the HLA-DRB1 associated SE. The mechanism for this effect is presumed to be because of differences in the ability of various GST enzymes to utilise the cytotoxic products of oxidant stress. Although significance was lost after correction for multiple testing, the data indicate that further studies may be of value in RA to determine the influence of the GST and other genes involved in cellular protection against oxidative stress. (Ann Rheum Dis 1999;58:164-168)

Rheumatoid arthritis (RA) is characterised by chronic inflammation of synovial joints with progressive destruction of cartilage and bone. The genetic factors that determine susceptibility and outcome are unclear though the HLA system, particularly the HLA-DRB1 molecule, is important..$^{1-3}$ This includes subtypes of DR4 $\left(\mathrm{DRB} 1{ }^{\star} 0401,{ }^{\star} 0404,{ }^{\star} 0405,{ }^{\star} 0408\right), \mathrm{DR} 1$ $\left(\mathrm{DRB} 1{ }^{\star} 0101,{ }^{\star} 0102\right)$ and $\mathrm{DRB} 1{ }^{\star} 1001$ and $\star 1402$. All the HLA-DRB1 associated alleles encode a conserved amino acid sequence (QKRAA, QRRAA or RRRAA) known as the shared epitope SE. ${ }^{4}$ Several studies indicate that certain combinations of SE carrying alleles are associated with more severe disease as measured by radiological outcome, or extra-articular manifestations, or both. ${ }^{35-7}$ Other susceptibility or outcome candidate genes for RA have yet to be identified.

Inflammation is a central feature of RA with the resulting reactive oxygen species (ROS) causing oxidation of DNA and lipids giving rise to a variety of cytotoxic products including lipid and DNA hydroperoxides and alkenals. ${ }^{8}$ There is evidence implicating ROS and their products in the pathology of RA. For example, ROS is produced by phagocytes in the synovial fluid and pannus and by synovial endothelial cells during hypoxia-reperfusion events. ${ }^{9}$ This suggests that variations in host effectiveness in the detoxification of products of ROS activity is important. Accordingly, we propose that polymorphism in enzymes that detoxify ROS and their products may contribute to the wide variation seen in the amount of joint damage and functional impairment.

The widely expressed, glutathione S-transferase (GST) supergene family seems to be critical in cellular protection against ROS. ${ }^{810}$ These enzymes catalyse the conjugation of glutathione with electrophiles such as lipid hydroperoxides, 4-hydroxy non-2-enal, DNA hydroperoxides. Polymorphism has been identified in several GST genes. Three alleles are described for GSTM1; GSTM $1{ }^{\star} 0$ is deleted while $G S T M 1^{\star} A$ and $G S T M{ }^{\star}{ }^{\star} B$ encode monomers that form active enzymes. ${ }^{8}$ GSTM3 is biallelic with $G S T M 3^{\star} A$ and $G S T M 3^{\star} B$, differing by a 3 base pair deletion in the latter that creates a motif for the YY1 transcription factor. 
Furthermore, $G S T M 3^{\star} B$ and $G S T M 1{ }^{\star} A$ are in linkage disequilibrium. A null polymorphism has also been identified at GSTT $1 .^{8}$ In the case of GSTP1, three alleles have been identified apart from the wild type $G S T P 1{ }^{\star} A$; one contains an A-G transition at nucleotide +313 $\left(G S T P 1{ }^{\star} B\right)$, one contains a C-T transition at +341 in addition to the A-G transition $\left(G S T P 1^{\star} C\right)$, while another contains only the C-T transition at $+341\left(G S T P 1^{\star} D\right)$. The +313 transition has been shown to result in altered catalytic activity, ${ }^{112}$ though there is no evidence to date of a functional effect of the +341 transition. Therefore we have studied only the +313 variant alleles. We have designated homozygotes for this transition, $G S T P 1^{\star} B /{ }^{\star} B$, though it is possible that a proportion of these will have the $G S T P 1{ }^{\star} C$ allele.

Support for the view that GST polymorphisms influence susceptibility/outcome to diseases with an inflammatory component comes from case control studies. Thus, GSTM1 null is associated with increased production of anti-Ro antibodies in patients with systemic lupus erythematosus and GSTT1 null confers increased risk of ulcerative colitis. ${ }^{8}$ Pilot data also suggest that particular GSTM1/GSTM3 genotype combinations confer increased susceptibility to oxidant stress. ${ }^{10}$ There are few data on the recently identified GSTP1 polymorphism. On the basis of in vitro and molecular epidemiological studies, particular genotypes can be considered high risk in the context of detoxification of ROS products. These are GSTM $1^{\star} 0 /{ }^{\star} 0,{ }^{810}$ GSTT $1^{\star} 0 /{ }^{\star} 0,{ }^{8} 10$ GSTM $3{ }^{\star} A /{ }^{\star} A,{ }^{10} G S T P 1{ }^{\star} B /{ }^{\star} B .{ }^{11}$ We now describe studies to determine if these putatively high risk genotypes are associated with susceptibility or outcome in RA.

\section{Methods}

GST GENOTYPES AND SUSCEPTIBLITY TO RA

The association between GST genotypes and susceptibility was studied in 277 unrelated northern European white RA patients resident in north Staffordshire (40.4\% male, median age 58.5, range 32.1-80 years) with a median disease duration of 11 years (range, 5-39). Of these, $60.5 \%$ were rheumatoid factor positive. They were recruited in a clinic established to examine the effects of slow acting antirheumatic drugs. Treatment was administered as clinically indicated. The ARA criteria of 1958 were recorded at the first presentation and have been used as the basic definition of the cohort. The 1987 ARA criteria were documented retrospectively from data in the case notes. The control group comprised 577 ethnically matched people (48\% male, mean age 70 years) suffering varicose veins, hernias, haemorrhoids, or mild iron deficiency anaemia. ${ }^{10}$ Patients with inflammatory disorders such as ulcerative colitis, diabetes or asthma were excluded.

GST, DISEASE OUTCOME, AND ACUTE PHASE RESPONSE

The association between disease outcome, acute phase response, and GST was studied in a subset of $122 \mathrm{RA}$ patients (38.5\% male, median age 58.1 years, range 32.1-79.0) selected as having a disease duration of $5-10$ years (median 8.3). This was to minimise the considerable between patient variation in disease duration (5-39 years) present in the total case group and to reduce the possible ceiling effect in radiological scores seen with increasing disease duration. ${ }^{13}$ These patients had been reviewed annually and their disease extensively characterised. Outcome measures were recorded at final review and consisted of assessment of functional status using the Health Assessment Questionnaire (HAQ) ${ }^{14}$ and radiographic outcome, obtained by scoring radiographs of the hands and feet using the standard radiographs of Larsen. ${ }^{15}$ The time integrated acute phase response was assessed by measuring erythrocyte sedimentation rate (ESR) and serum $\mathrm{C}$ reactive protein (CRP) concentrations at yearly intervals for at least five years and calculating the area under the curve (AUC) for these parameters. Division of the AUC by the number of years follow up gave the mean area under the curve (MAUC) ${ }^{16}$

GST AND HLA-DR GENOTYPING

Genotypes were identified from lymphocyte DNA. GSTM1 genotypes were defined using a PCR assay that identifies $G S T M 1{ }^{\star} 0$ homozygotes, GSTM $1^{\star} A{ }^{\star} B$ heterozygotes and the GSTM1 A and GSTM1 B phenotypes. It does not distinguish $G S T M 1{ }^{\star} 0 /{ }^{\star} A$ and $G S T M 1{ }^{\star} A /$ ${ }^{\star} A$ genotypes or the equivalent GSTM1 $\mathrm{B}$ genotypes. ${ }^{8}$ The GSTM $3{ }^{\star} A /{ }^{\star} A,{ }^{\star} A /{ }^{\star} B$ and ${ }^{\star} B /{ }^{\star} B$ genotypes were identified by amplifying the exon 6/7 regions of GSTM3 and differentiating $G S T M 3^{\star} A$ from $G S T M 3^{\star} B$ by digestion with $M n I I .{ }^{8}$ The GSTP1 ${ }^{\star} A /{ }^{\star} A,{ }^{\star} A /{ }^{\star} B$ and ${ }^{\star} B /{ }^{\star} B$ genotypes were identified using primers to exon $5 .{ }^{10}$ The $G S T T 1^{\star} 0$ and expressing subjects were also identified using poymerase chain reaction. ${ }^{8}$ Presence of the HLA-DRB1 SE was identified in people using methods previously described. ${ }^{17} 18$

\section{STATISTICAL ANALYSIS}

Genotype frequencies were compared between cases and controls using $\chi^{2}$ tests (StatXact Turbo). Disease severity and activity variables were first transformed to normality, where appropriate, and the effects of genotypes were assessed using multiple regression analysis with the addition of the independent variables, age, sex, disease duration, SE, and rheumatoid factor (RF) positivity where these were significant. Correction for potential multiple testing errors was performed using Holm's procedure. ${ }^{19}$ This is less conservative than the widely used Bonferroni procedure and has been considered as being uniformly better. ${ }^{20}$ In each set of analyses six tests were performed (GSTM1, GSTT1, GSTM3, GSTP1, GSTM1+GSTM3, GSTM1 +GSTT1).

\section{Results}

CASE CONTROL STUDY: GST AND SUSCEPTIBLITY The clinical indices of the total group of 277 RA patients, and the 122 patients with disease duration 5-10 years were similar to other studies, and there was typical heterogeneity in 
terms of age of onset and disease duration. Table 1 summarises GST genotype frequencies. Differences were identified for GSTM1; the frequency of $G S T M 1^{\star} A{ }^{\star} B$ in the cases was significantly lower than in the controls $\left(\chi_{1}^{2}=4.7\right.$, exact $\mathrm{p}=0.047$, odds ratio $0.28,95 \%$ CI 0.054, 0.962). However, this was not significant after correction for potential multiple testing errors. The frequency of GSTM $3^{\star} B /{ }^{\star} B$ was lower in the cases though the difference did not achieve significance (exact $\mathrm{p}=0.12$ ). No significant differences in the frequencies of GSTT1 or GSTP1 genotypes were identified.

ASSOCIATION OF GST GENOTYPES WITH MARKERS OF DISEASE OUTCOME AND ACUTE PHASE RESPONSE

Larsen scores together with HAQ, MAUCESR and MAUCCRP data from these patients are shown in table 2. Comparisons were made between individual GST genotypes, and also between putatively high risk genotypes and the corresponding complement (that is, GSTM1 null compared with non-null genotypes).

GSTM1

The highest mean Larsen score (89.9) was found in patients with $G S T M 1^{\star} 0 /{ }^{\star} 0$, which

Table 1 GST genotypes in total RA case group and controls

\begin{tabular}{|c|c|c|c|c|}
\hline & GSTM1 null & ${ }^{\star} A$ & ${ }^{\star} B$ & ${ }^{\star} A /{ }^{\star} B$ \\
\hline Controls $(n=591)$ & $345(58.4 \%)$ & $152(25.7 \%)$ & $72(12.2 \%)$ & $22(3.7 \%)$ \\
\hline \multirow[t]{2}{*}{ RA cases $(n=276)$} & $152(55.1 \%)$ & $76(27.5 \%)$ & $45(16.3 \%)$ & $3(1.1 \%)^{\star}$ \\
\hline & $\mathrm{GSTM} 3^{\star} \mathrm{A} /{ }^{\star} \mathrm{A}$ & ${ }^{\star} \mathrm{A} /{ }^{\star} \mathrm{B}$ & ${ }^{\star} \mathrm{B} /{ }^{\star} \mathrm{B}$ & \\
\hline Controls $(n=295)$ & $221(74.9 \%)$ & $59(20.0 \%)$ & $15(5.1 \%)$ & \\
\hline \multirow[t]{2}{*}{ RA cases $(n=254)$} & $186(73.2 \%)$ & $62(24.4 \%)$ & $6(2.4 \%)$ & \\
\hline & GSTT1 null & GSTT $1{ }^{\star} \mathrm{A}$ & & \\
\hline Controls $(n=556)$ & $451(81.1 \%)$ & $105(18.9 \%)$ & & \\
\hline \multirow[t]{2}{*}{ RA cases $(n=275)$} & $223(81.1 \%)$ & $52(18.9 \%)$ & & \\
\hline & $\mathrm{GSTP} 1{ }^{\star} \mathrm{A} /{ }^{\star} \mathrm{A}$ & $\mathrm{GSTP} 1{ }^{\star} \mathrm{A} /{ }^{\star} \mathrm{B}$ & $\mathrm{GSTP} 1{ }^{\star} \mathrm{B} /{ }^{\star} \mathrm{B}$ & \\
\hline Controls $(n=297)$ & $136(45.8 \%)$ & $118(39.7 \%)$ & $43(14.5 \%)$ & \\
\hline RA cases $(n=226)$ & $91(40.3 \%)$ & $104(46.0 \%)$ & $31(13.7 \%)$ & \\
\hline
\end{tabular}

${ }^{\star}$ Frequency of GSTM $1{ }^{\star} \mathrm{A} /{ }^{\star} \mathrm{B}$ in cases and controls: exact $\mathrm{p}=0.047, \chi^{2}=4.7$, OR $0.28,95 \% \mathrm{CI}$ $0.054,0.962$. (Not significant after correction for potential multiple testing errors).

Table 2 GST genotypes and measures of disease outcome and activity in 122 RA patients with disease duration 5-10 years

\begin{tabular}{|c|c|c|c|c|c|}
\hline Genotype & Number & $\begin{array}{l}\text { Larsen score } \\
(S D)\end{array}$ & $\begin{array}{l}H A Q \\
(S D)\end{array}$ & $\begin{array}{l}\text { MAUCESR } \\
(S D)\end{array}$ & $\begin{array}{l}\text { MAUCCRP } \\
(S D)\end{array}$ \\
\hline GSTM1 null & 63 & $89.9(43.3)$ & $1.33(0.82)$ & $27.9(18.0)$ & $17.1(14.6)$ \\
\hline GSTM1*A & 42 & $70.7(42.8)$ & $1.22(0.86)$ & $24.3(13.7)$ & $15.1(16.9)$ \\
\hline GSTM $1 \star B$ & 15 & $83.2(32.3)$ & $1.67(0.91)$ & $30.4(10.7)$ & $20.8(17.8)$ \\
\hline $\mathrm{GSTM} 1{ }^{\star} \mathrm{A} /{ }^{\star} \mathrm{B}$ & 1 & 115.0 & 1.80 & 46.9 & ND \\
\hline GSTM1 non-null & 58 & $74.7(40.4)^{\star}$ & $1.39(0.89)$ & $26.3(13.3)$ & $16.8(17.1)$ \\
\hline $\mathrm{GSTM}^{\star} 3^{\star} \mathrm{A} /{ }^{\star} \mathrm{A}$ & 86 & $86.3(43.3)$ & $1.34(0.86)$ & $27.9(17.3)$ & $17.8(17.1)$ \\
\hline GSTM $3{ }^{\star} \mathrm{A} /{ }^{\star} \mathrm{B}$ & 23 & $77.3(40.9)$ & $1.18(1.18)$ & $25.0(12.4)$ & $15.8(14.8)$ \\
\hline GSTM $3{ }^{\star} \mathrm{B} /{ }^{\star} \mathrm{B}$ & 3 & $98.3(30.9)$ & $1.33(1.18)$ & $23.2(13.8)$ & $21.8(20.1)$ \\
\hline GSTM3 non ${ }^{\star} \mathrm{A} /{ }^{\star} \mathrm{A}$ & 26 & $79.7(40.0)$ & $1.19(0.86)$ & $24.8(12.3)$ & $16.4(14.1)$ \\
\hline GSTT1 null & 28 & $77.9(33.4)$ & $1.54(0.87)$ & $30.8(18.8)$ & $20.3(21.3)$ \\
\hline $\mathrm{GSTT} 1{ }^{\star} \mathrm{A} /{ }^{\star} \mathrm{A}$ & 93 & $84.0(44.9)$ & $1.28(0.84)$ & $26.0(14.8)$ & $15.9(13.7)$ \\
\hline $\mathrm{GSTP} 1{ }^{\star} \mathrm{A} /{ }^{\star} \mathrm{A}$ & 41 & $82.3(39.37)$ & $1.45(0.92)$ & $23.7(15.5)$ & $15.8(14.4)$ \\
\hline $\mathrm{GSTP} 1{ }^{\star} \mathrm{A} /{ }^{\star} \mathrm{B}$ & 44 & $82.7(46.0)$ & $1.21(0.86)$ & $30.0(17.6)$ & $18.1(18.7)$ \\
\hline $\mathrm{GSTP} 1{ }^{\star} \mathrm{B} /{ }^{\star} \mathrm{B}$ & 10 & $107.1(35.4)$ & $1.52(0.70)$ & $32.0(16.8)$ & $21.4(18.4)$ \\
\hline GSTP1 non ${ }^{\star} \mathrm{B} /{ }^{\star} \mathrm{B}$ & 85 & $82.5(42.5)$ & $1.27(0.87)$ & $27.2(16.6)$ & $16.9(16.7)$ \\
\hline
\end{tabular}

${ }^{\star} \mathrm{p}=0.039$ (compared with GSTM1 null, after correction for age, sex, and disease duration). ND $=$ not done.

Table 3 Association between HLA-DRB1 SE and disease measures in RA patients with disease duration 5-10 years

\begin{tabular}{llllll}
\hline Genotype & Number & $\begin{array}{l}\text { Larsen score } \\
(S D)\end{array}$ & $\begin{array}{l}\text { HAQ } \\
(S D)\end{array}$ & $\begin{array}{l}\text { MAUCESR } \\
(S D)\end{array}$ & $\begin{array}{l}\text { MAUCCRP } \\
(S D)\end{array}$ \\
\hline SE +/+ & 39 & $87.9(38.4)$ & $1.33(0.80)$ & $25.7(13.8)$ & $16.8(14.9)$ \\
SE +/- & 51 & $79.3(39.1)$ & $1.37(0.88)$ & $26.6(16.1)$ & $17.3(15.0)$ \\
SE -/- & 27 & $74.3(51.2)$ & $1.13(0.84)$ & $27.4(16.9)$ & $16.2(18.8)$ \\
\hline
\end{tabular}

was significantly different $(\mathrm{p}=0.039)$ to nonnull people (74.7) after adjustment for age, sex, and disease duration. Correction for the presence of the SE did not change the association of Larsen score with $G S T M 1{ }^{\star} 0 /{ }^{\star} 0$ $(\mathrm{p}=0.045)$. Furthermore, this association was not confounded by RF positivity as addition of this variable to the multiple regression analysis had little impact $(p=0.031)$. Adjustment for possible mutiple testing errors resulted in loss of significance. The lower Larsen score in nonnull people seemed to be accounted for largely by people with the GSTM1 A phenotype (70.7) although the difference from GSTM1 B people (83.2) was not significant $(\mathrm{p}=0.6)$ after correction for age, sex, and disease duration. No significant associations between GSTM1 genotypes and the age of onset, HAQ score or, measures of the acute phase response were identified.

\section{GSTM3 and GSTT1}

No significant associations between GSTM3 or GSTT1 genotypes and Larsen score, HAQ, MAUCESR or MAUCCRP were seen. Furthermore, no interactions between GSTM1 null and GSTT1 null or between GSTM1 null and $G S T M 3^{\star} A /{ }^{\star} A$ were identified.

\section{GSTP1}

The differences in Larsen scores between individual GSTP1 genotypes were not significant, although an association between the GSTP1 ${ }^{\star} B /{ }^{\star} B$ and the Larsen score approached significance $(\mathrm{p}=0.096$; corrected for $\mathrm{SE}$, $\mathrm{p}=0.064)$ when compared with non $-{ }^{\star} B /{ }^{\star} B$ people. Additional correction for RF positivity as well as the SE achieved a significant difference $(p=0.04)$, though significance was lost after adjusment for multiple testing. There were no associations between GSTP1 genotypes and age of onset, HAQ, MAUCCRP or MAUCESR, with or without correction for the SE and RF positivity.

COMPARISON WITH THE SE

As the presence of the SE has been associated with more severe disease we examined the association of the SE with markers of outcome and acute phase response, for comparison with the GST genotypes. The highest Larsen score (87.9) was found in SE homozygous patients, and the lowest (74.3) in patients negative for the SE (table 3). Although not statistically significant $(p=0.14$, after correction for age, sex, and disease duration) the trend was consistent with previous studies. Interestingly the Larsen score for the SE homozygous patients (87.9) was similar to that for GSTM1 null patients (89.9), while the score for SE negative people (74.3) was essentially the same as that for patients with a positive GSTM1 allele (74.7). No significant differences were found between SE homozygous and SE negative patients for age of onset, HAQ, MAUCESR and MAUCCRP.

Correction for the presence of the SE in our multiple regression analysis indicated that it had little influence on the difference in Larsen scores between GSTM1 null and non-null patients. This is illustrated clearly in table 4 
Table 4 Comparison of Larsen scores in GSTM1 null and non-null patients according to SE dose

\begin{tabular}{llllll}
\hline & \multicolumn{2}{l}{ GSTM1 null } & & \multicolumn{2}{l}{ GSTM1 non-null } \\
\cline { 2 - 3 } \cline { 6 - 6 } Genotype & Number (\%) & Larsen (SD) & & Number (\%) & Larsen (SD) \\
\hline SE +/+ & $23(38.3)$ & $94.3(42.4)$ & & $16(28.1)$ & $78.7(30.5)$ \\
SE +/- & $26(43.3)$ & $81.1(39.6)$ & & $25(43.8)$ & $79.9(39.4)$ \\
SE -/- & $11(18.3)$ & $91.8(42.4)$ & & $16(28.1)$ & $58.7(49.1)$ \\
\hline
\end{tabular}

where GSTM1 null and non-null patients have been divided into $\mathrm{SE}+/+, \mathrm{SE}+/-$, and $\mathrm{SE}-/-$ groups. The frequency of SE-/- and SE+/+ people is not significantly different between GSTM1 null and non-null groups, and in GSTM1 null patients no statistical differences in Larsen score was found between patients with or without the SE. In non-null patients the difference between the groups was not significant although the trend suggested a lower Larsen score in SE-/- patients.

\section{Discussion}

We have proposed that GSTM1, GSTM3, GSTT1, and GSTP1 are candidate genes for susceptibility and outcome in RA. We used a case-control approach to identify associations between GST genotypes and susceptibility to RA. Our purpose was exploratory and we realise there are dangers in making inferences on individual factors in multiple testing. Results are considered as hypothesis setting and needing confirmation in further studies. Our data suggest that GSTM1 ${ }^{\star} A{ }^{\star} B$, but not the GSTM1 A or GSTM1 B phenotypes, may be associated with reduced susceptibility. This indicates a gene dose effect; thus, GSTM1 ${ }^{\star} A{ }^{\star} B$ is protective because most people defined as GSTM1 A or GSTM1 B phenotypes are $G S T M 1{ }^{\star} 0 /{ }^{\star} A$ and $M 1^{\star} 0 /{ }^{\star} B$ heterozygotes respectively. ${ }^{8}$ These data and others reporting that the genotype is protective against other oxidative stress related diseases such as basal cell carcinoma suggest that GSTM1 is important in cellular protection, though the mechanism is unclear. ${ }^{8}$ Given the reported linkage disequilibrium with GSTM3 alleles, it is possible that the biological effect of GSTM1 is exerted through other mu class GST or another neighbouring gene. In our particular RA population the extent of linkage between GSTM1 and GSTM3 was no different to that in controls (data not shown).

We also assessed the influence of GST polymorphisms on outcome and the acute phase response in RA. A more severe radiological outcome, was associated with GSTM1 null, while the association with GSTP1 ${ }^{\star} B /{ }^{\star} B$ approached significance. The association was independent of RF positivity and the presence of the SE, both of which have been associated with more severe disease..$^{521-23}$ Interestingly the Larsen scores in GSTM1 null and $G S T P 1{ }^{\star} B /{ }^{\star} B$ patients were equivalent or greater than Larsen scores for patients with a double dose of the SE. A small but nonsignificant increase in Larsen score was seen in patients with both GSTM1 null and SE+/+. This preliminary study suggests that the GSTM1 null genotype may be an independent marker for development of more erosive disease. However, the association seems to be weak and did not achieve significance after correction for potential multiple testing errors. Thus its clinical usefulness may be limited and further studies are needed to determine more clearly the significance of this finding.

We did not identify any associations between GST genotypes and functional outcome assessed using the HAQ score. This may partly be explained by the fact that such scores include factors like pain perception, neuromuscular power and psychosocial factors that are difficult to assess precisely and may therefore, be difficult to correlate with single genotypes. Other factors may also cause discrepancy between radiologically assessed and functional outcomes. For example, Larsen score primarily assesses damage to cartilage and bone but does not necessarily reflect damage to other tissues and organs. Thus, in the joint, unknown levels of damage to tendons, ligaments and soft tissue, together with neurological changes and muscle wasting will all be important in determining clinical and functional outcome.

Previous studies have shown that measures of the acute phase response over 5-10 years are significantly correlated with radiological assessment of joint deterioration, and that persistently raised ESR and CRP values are associated with more radiological progression. ${ }^{16}$ However, there is also evidence that erosion and inflammatory response may follow diverging paths. ${ }^{24}$ In this study no associations between GST genotypes and MAUCCRP or MAUCESR were seen. We interpret this as indicating that the inflammatory response is not dependent on GST genotype, it being more probable that the GSTs serve to modify the effects of inflammation. Thus the radiological outcome in people with the same amount of inflammation over time may be different depending on GST status. Although one might expect to see a general association beween inflammatory markers and radiological outcome it would not necessarily be seen between these markers and the GST genotypes. The same applies to the DRB1 SE where the relation with the inflammatory response and radiological outcome is still far from clear.

Our results suggest that genes involved in protection from oxidant stress may influence the disease process in RA. The influence of these genotypes may contribute with other genes that exert a relatively weak influence. ${ }^{8}$ Though such weak effects may be real they tend to disappear when adjustment for multiple testing is carried out. It is unclear which GST substrates are important and why certain GST seem influential in some but not all diseases in which oxidant stress is a critical feature. This may reflect tissue specific patterns of gene expression. GSTP1 is of interest as it is widely expressed in human cells including synovial fibroblasts and lymphocytes. ${ }^{25}$ In contrast, expression of GSTM1 protein is more restricted, being found in lymphocytes but not in synovial fibroblasts. ${ }^{25}$ 
We gratefully acknowledge the generous support of the Arthritis Research Campaign, the Haywood Rheumatism Research and Development Foundation, the European Commission (conClarke and June Fisher, metrologists at the Staffordshire RheuClarke and June
matology Centre.

1 Gao X, Olsen NJ, Pincus T, Stastny P. HLA-DR alleles with naturally occuring amino acid substitutions and risk for development of rheumatoid arthritis. Arthritis Rheum 1990;30:1205-13.

2 Thomson W, Pepper L, Payton T, Carthy D, Scott D, Ollier $\mathrm{W}$, et al. Absence of an association between HLA DRB ${ }^{\star} 04$ and RA in newly diagnosed cases from the community. Ann Rheum Dis 1993;52:539-41.

3 MacGregor A, Ollier W, Thomson W, Jawaheer D, Silman A. HLA-DRB ${ }^{\star} 0401 / 0404$ genotype and rheumatoid arthritis: Increased association in men, young age at onset and disease severity. J Rheumatatol 1995;22:1032-6.

4 Gregersen PK, Silver J, Winchester RJ. The shared epitope hypothesis: An approach to understanding the molecular genetics of susceptiblity to rheumatoid arthritis. Arthritis Rheum 1987;30:1205-13.

5 Moreno I, Valenzuela A, Garcia A, Yelarnos J, Sanchez B, Moreno I, Valenzuela A, Garcia A, Yelarnos J, Sanchez B, Hernanz W. Association of the shared epitope with
radiological severity of rheumatoid arthritis. J Rheumatol 1996;23:6-9.

6 Weyand CM, Xie C, Goronzy JJ. Homozygosity for the HLA-DRB1 allele selects for extraarticular manifestation in rheumatoid arthritis. J Clin Invest 1992;89:2033-9.

7 Perdriger A, Chales G, Semana G, Guggenbuhl P, Meyer O, Quillivic F, et al. Role of HLA-DR-DR and DR-DQ associations in the expression of extraarticular manifestations and rheumatoid factor in rheumatoid arthritis. J Rheumatol 1997;24:1272-6.

8 Strange RC, Fryer AA. The glutathione S-transferases: influence of polymorphism on susceptibility to cancer. In: Boffetta P, Caporaso N, Cuzick J, Lang M, Vineis P, eds. Metabolic polymorphisms and cancer (in press).

9 Blake DR, Merry P, Unsworth J, Kidd BI, Outhwaite JM, Ballard R, et al. Hypoxia-reperfusion injury in the inflammed human joint. Lancet 1989;i:289-93.

10 Yengi L, Inskip A, Gilford J, Alldersea J, Bailey L, Smith A, et al. Polymorphism at the glutathione S-transferase et al. Polymorphism at the glutathione S-transferase GSTM3 locus: Interactions with cytochrome P450 and glutathione S-transferase genotypes as risk factors for mu
tiple basal cell carcinoma. Cancer Res 1996;56:1974-7.

11 Ali-Osman F, Akande O, Antoun G, Mao J-X, Buolamwin J. Molecular cloning, characterization and expression in Escherichia coli of full-length cDNAs of three human glutathione S-transferase Pi gene variants. J Biol Chem 1997;272:10004-12.
12 Harries LW, Stubbins MJ, Forman D, Howard GCW, Wolf CR. Identification of genetic polymorphism at the GSTP1 locus and association with susceptibility to bladder, testicular and prostate cancer. Carcinogenesis 1997;18: $641-4$.

13 Sharp JT. Attempts to apply curve fitting models to the analysis of progression of radiographic damage in rheumatoid arthritis are laudable, but are the results believable? J Rheumatol 1994;21:1589-90.

14 Fries JF, Spitz P, Kraines RG, Holman HR. Measurement of patient outcome in arthritis. Arthritis Rheum 1980;23: $137-45$.

15 Larsen A, Dale K, Eck M. Radiographic evaluation of rheumatoid arthitis and related conditions by standard eference films. Acta Radiol (Diagn Stockh) 1977;18:48191 .

16 Hassell AB, Davis MJ, Fowler PD, Clarke S, Fisher J, Shadforth MF, et al. The relationship between serial measures of disease activity and outcome in rheumatoid arthritis. Q J Med 1993;86:601-7.

17 Thomson W, Ollier WER. Non-radioactive ASO typing for class II. Eur J Immunogenet 1992;19:169-75.

18 Jawaheer D, Ollier WER, Thomson W. Multiplex ARMSRFLP: a rapid and simple method for HLA-DR4 RFLP: a rapid and simple method for H
subtyping. Eur J Immunogenet 1993;20:279-91.

19 Holm S. A simple sequentially rejective multiple test procedure. Scandinavian Journal of Statistics 1979;6:65-70.

20 Aickin M, Gensler H. Adjusting for multiple testing when reporting research results: the Bonferroni vs Holm methods. Am J Public Health 1996;86:726-8.

21 Weyand CM, Hicok KC, Conn D, Goronzy JJ. The influence of HLA-DRB1 genes on disease severity in rheumatoid arthritis. Ann Intern Med 1992;117:801-6.

22 Wordsworth P, Pile KD, Buckley JD, Lanchbury JSS, Ollier $\mathrm{B}$, Lathrop M, et al. HLA heterozygosity contributes to $\mathrm{B}$, Lathrop $\mathrm{M}$, et al. HLA heterozygosity contributes to
susceptibility to rheumatoid arthritis. Am J Hum Genet susceptibility to

23 Weyand CM, McCarthy TG, Goronzy JJ. Correlation between disease phenotype and genetic heterogeneity in rheumatoid arthritis. J Clin Invest 1995;95:2120-6.

24 Mulherin D, Fitzgerald O, Bresnihan B. Clinical improvement and radiological deterioration in rheumatoid arthritis: evidence that the pathogenesis of synovial inflammation and articular erosion may differ. $\mathrm{Br} J$ Rheumatol 1996;35:1263-8.

25 Mattey DL, Nixon N, Alldersea JE, Cotton W, Fryer AA, Zhao L, et al. Alpha, mu and pi class glutathione S-transferases in human synovium and cultured synovial fibroblasts: effects of interleukin-1a, hydrogen peroxide and inhibition of eicosanoid synthesis. Free Radic Res Commun 1993;19:159-71 